\title{
Percepción del profesorado de Educación Física sobre el Aprendizaje Cooperativo y su relación con la Inteligencia Emocional Physical Education teacher's perception of Cooperative Learning and its relation to Emotional Intelligence
}

\author{
Adela Sánchez-M olina, Irene González-Martí, Andrea Hernández-Martínez \\ Universidad de CastillaLLa Mancha (España)
}

\begin{abstract}
Resumen. El presente trabajo trata uno de los aspectos más estudiados en Educación Física como es el modelo pedagógico deA prendizaje Cooperativo y su repercusión sobre el alumnado en edad escolar. El objetivo principal de esta investigación fue conocer la percepción del profesorado del área de Educación Física en la etapa de Educación Primaria acerca del uso del A prendizaje Cooperativo y su rel ación con la InteligenciaEmocional. Paraello, sellevó a cabo unainvestigación cual itativa por medio de entrevistas semiestructuradas a través de la opinión de un total de seis maestros de Educación Física pertenecientes a la etapa educativa de Educación Primaria. Los resultados obtenidos confirman que los docentes muestran una actitud positiva y poseen buenas percepciones hacia este modelo pedagógico. Sin embargo, todos coinciden en el argumento de que se necesita mucho esfuerzo y trabajo, así como seguimiento constante para obtener beneficios y resultados significativos. Asimismo, afirman que este modelo está estrechamente relacionado con la Inteligencia Emocional, considerándolo un aspecto que lo enriquece todavía más.
\end{abstract}

Palabras clave: Percepción profesorado, Educación Física, A prendizaje Cooperativo, Inteligencia Emocional.

\begin{abstract}
The present work deals with one of the most studied aspects of Physical Education such as the pedagogical model of Cooperative Learning and its impact on shool-age students. The main goal of this research was to know the perception of teachers in the area of Physical Education in Primary Education about the use of Cooperative Learning and its relationship with Emotional Intelligence. To this end, qualitative research was carried out through semi-structured interviews thanks to the opinion of a total six Physical Education teachers from the Primary Education. The results obtained confirm that teachers show a positive attitude and have good perceptions towards this pedagogical model. However, everyone agrees on the argument that it takes a lot of effort and work, as well as constant monitoring to obtain benefits and significative results. They al so affirm that this model is closely related to Emotional Intelligence, considering it as an aspect that enriches it even more. Key words:Teachers perception, Physical Education, Cooperative Learning, Emotional Intelligence.
\end{abstract}

\section{Introducción}

El Real Decreto 126/ 2014, por el que se establece el currículo básico de Educación Primaria, indica que el área de Educación Física (EF) tiene como finalidad principal el desarrollo de la competencia motriz del alumnado a través de la integración de conocimientos, procedimientos, actitudes y sentimientos relacionados con la conducta motriz. Sin embargo, para su consecución es fundamental un análisis crítico que afiance actitudes y valores vinculados al cuerpo y al movimiento, permitiendo dar sentido a las propias acciones motrices, así como comprender aspectos perceptivos, emotivos, y cognitivos de las mimas.

Como consecuencia, y de acuerdo con FernándezRío, Calderón, Hortigüela, Pérez-Pueyo y Aznar (2016),

Fecha recepción: 22-11-20. Fecha de aceptación: 04-02-21

Andrea Hernández Martínez

andrea.hernandez@uclm.es cada vez son más los docentes de EF que incorporan en sus clases la enseñanza basada en modelos pedagógicos, entendidos estos como aquellos model os que se centran en el aprendizaje del alumno, resaltando la interdependencia del aprendizaje, la enseñanza, el contenido y el contexto (Rovegno, 2006), buscando de esta manera una experiencia auténtica y enriquecedora para estos.

Uno de los modelos pedagógicos más estudiados e implementados en el área de EF, es el Aprendizaje Cooperativo ( $\mathrm{AC}$ ). En este modelo los alumnos trabajan juntos, en pequeños grupos heterogéneos, y aprenden con y de sus compañeros mediante un planteamiento de enseñanza-aprendizaje que fomenta la interacción e interdependencia positivas, permitiendo al docente y a losestudiantes actuar como co-aprendices (Dyson, 2001; Fernández-Río, 2014). Asimismo, se cita en el Decreto 54/ 2014, por el que se establece el currículo de Educa ción Primaria en la Comunidad Autónoma de CastillaLaM ancha, y en concreto en el área de EF, como uno de los modelos pedagógicos más significativos y adecuados 
para el desarrollo integral y moral del alumnado, ya que favorece el aprendizaje y la adquisición de valores, permitiendo formar individuos capaces de convivir y relacionarse en sociedad.

Igualmente, cabe destacar que su uso adecuado ofrece la posibilidad al alumnado de aprender habilidades sociales, aumentar la interacción entre iguales, mejorar la capacidad de comunicarse y tomar decisiones conjuntamente, favorecer el desarrollo de la interdependencia, la interculturalidad, la motivación, aprender pautas paralaresolución de conflictos(Velázquez, 2010), pero sobre todo, les ayuda a desarrollar una actitud positiva hacia el proceso de enseñanza-aprendizaje, y especialmente les enseña a valorar la clase de EF (Fernández-Río, 2003). No obstante, su comprensión teórica y su puesta en práctica no es tarea fácil, y requiere mucho esfuerzo y trabajo, así como formación y dominio por parte del docente. Como indican Fernández-Río, Hortigüela y Pérez-Pueyo (2018), Pérez-Pueyo y Hortigüela (2019) y Velázquez (2015), son muchos los maestros y maestras de EF que afirman implementar el modelo pedagógico deAC en sus clases porque utilizan juegos cooperativos de forma puntual, 0 simplemente ponen en práctica estrategias de trabajo en grupo. De este modo, queda reflejado el desconocimiento de esta metodología por parte de muchos docentes quienes confunden los términos de trabajo en grupo, colaboración y cooperación (Fernández-Río, Rodríguez, Velázquez y Santos, 2014).

En consecuencia, su uso incorrecto genera en el alumnado sentimientos y actitudes negativas y antisociales, que más que favorecer su desarrollo moral eintegral, generan situacionesy actitudes adver sas como pueden ser crisis de valores, fracaso escolar y acoso 0 abuso en diversas sustancias. Por este motivo, Johnson y Johnson (1999), concluyen que, para evitar situaciones negativas y, por tanto, la condición de AC sea real, es necesaria la presencia de una serie de condiciones mediadoras que conforman los componentes esencial es del mismo. Dichos componentes son: interdependencia positiva, interacción promotora (preferiblemente cara a cara), responsabilidad personal e individual, habilidades interpersonales y de grupo y, por último, procesamiento grupal o autoevaluación (Johnson y J ohnson, 1999). En definitiva, el AC es un modelo pedagógico con enormes posibilidades en el ámbito de la EF, pero para obtener beneficios significativos, es imprescindible que estén integrados todos sus componentes, a la vez que esté adaptado a las características e intereses de los alumnos y alumnas (Fernández-Río y Méndez-Giménez, 2016).
Puesto que se persigue una formación integral del alumnado, es necesario considerar el trabajo de las emociones como uno de los aspectos fundamentales, ya que el $A C$ ofrece oportunidades excepcional es para desarrollar la Inteligencia Emocional, tanto de manera individual como grupal (Bisquerra, Pérez y García, 2015). El aprendizaje en el aula depende en ocasiones más de la emoción que se genere en el alumnado que del motivo con el que se trabajen los objetivos de aprendizaje (García, 2012), por lo que al go que no motiva, no se aprende de manera tan sencilla. En este sentido, destaca la Inteligencia Emocional, definida por Goleman (1996) como la capacidad de reconocer tanto los propios sentimientos como los de los demás, de motivarse y desarrollar de manera adecuada las relaciones. Sin embargo, este concepto se corresponde más con el área de lapsicología, mientrasque en educación se hace mayor hincapiéen el concepto de competenciaemocional. Según Bisquerra y Pérez (2007), las competencias emociona les hacen referencia a un conjunto de conocimientos, capacidades, habilidades y actitudesnecesarias para comprender, expresar y regular de manera apropiada los fenómenos emocionales. Además, son un aspecto importante de la ciudadanía efectiva y responsable, potenciando una mejor adaptación al contexto y afrontando las circunstancias de la vida con mayor posibilidad de éxito. De esta manera, deberá ser estimulada y desa rrollada para que el alumno se conozca y respete, tanto a sí mismo como a los demás, y alcance el desarrollo completo desu personalidad (García, 2012). Como apunta Barrientos (2016), una persona inteligente emocionalmente es aquella que sabe convivir con las emociones o sentimientos desagradables, es capaz de transformarlosen agradables, utilizarlos de maneraefectiva y valorarlos como oportunidades de aprendizaje, cualidades que entendemos son esenciales para la vida.

Por todos los motivos previamente descritos, el objetivo principal de esta investigación fue conocer la percepción que posee el profesorado del área de EF, perteneciente alaetapaeducativa deEducación Primaria(EP), sobre el uso del modelo pedagógico deAC y su relación con la Inteligencia Emocional.

\section{Método}

El carácter de la investigación fue cualitativo, con un diseño no probabilístico, con muestreo por conveniencia. Los criterios de selección de los participantes fueron que impartiesen clases de EF en EP y que conociesen o utilizasen el modelo pedagógico de AC. 


\section{Participantes}

La muestra estuvo compuesta por seis maestros y maestras de $E F$, cuatro hombres y dos mujeres $(66,7 \%$ y $33,3 \%$ ), con un rango de edad entre 28 y 60 años (M $=41,5$ años). Dichos participantes contaban con una experiencia docente media de 18,6 años. La totalidad de la muestra impartía clases en centros públicos, cua tro situados en un entorno rural y dos en un entorno urbano $(66,7 \%$ y $33,3 \%$ respectivamente). Asimismo, habiendo trabajado anteriormente en una media de 4,5 centros escolares en el nivel de EP.

\section{Instrumentos}

El instrumento utilizado para la recogida de datos fue una entrevista semiestructurada compuesta por 30 preguntas diseñada para la presente investigación, donde se recogía información demográfica (5 preguntas; e. g. ¿cuánto tiempo lleva dedicándose a la enseñanza?) cuestiones referentes a la percepción del uso del modelo pedagógico de AC (7 preguntas; e.g. ¿cuáles son las técnicas de Aprendizaje Cooperativo que más le gustan?), consecuencias de su implementación (10 preguntas, iconside ra que su uso en las aulas es positivo para el alumnado?) y la relación de dicho modelo con la Inteligencia Emocional (8 preguntas; e.g. ipiensa que esta metodología de trabajo está estrechamente relacionada con la inteligencia emocional del alumnado?).

\section{Procedimiento}

Tras valorar que los participantes cumplieran los requisitos para poder formar parte de esta investiga ción, se contactó con los mismos vía telefónica. Antes de comenzar con la entrevista se les informó sobre el objetivo del estudio y del carácter anónimo y volunta rio de la participación, además de que en cualquier momento podría abandonar la entrevista. Tras obtener los correspondientes consentimientos, se concretaron citas para la realización de las entrevistas individuales, las cuales se llevaron a cabo mediante videollamadas a través de la plataforma M icrosoft Teams, con una duración media de 20 minutos.

\section{Análisis de datos}

Una vez finalizadas las transcripciones de las entrevistas, se realizó una lectura exhaustiva para obtener una comprensión global del contenido. A continuación, se codificaron y categorizaron a través de un análisis narrativo de contenido categorial (Pérez, Fuentes y Devís, 2011), mediante un método inductivo en el que emergieron distintas categorías o dimensiones.
En relación con la consistencia de los hallazgos se real izó la triangulación entre investigadores, todos ellos de la U niversidad de Castilla-La M ancha, miembros del grupo de investigación «Enseñanza del Deporte y la Actividad Física» (EDAF). De esta manera se pudieron contrastar opiniones y alcanzar soluciones e interpreta ciones consensuadas, tanto en el establecimiento de categorías de codificación como en la interpretación de los resultados. Los datos fueron analizados a través de los programas ATLAS.ti 8 y ATLAS.ti Cloud.

\section{Resultados y Discusión}

Un total de 330 citas repartidas en 33 códigos se determinaron en los análisis de las diferentes entrevistas. Dichas citas se agruparon en dimensiones, que apa recen recogidas en la siguiente tabla.

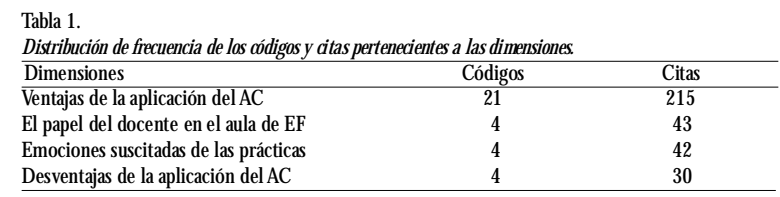

La dimensión que más códigos recoge es la de Ventajas de la aplicación del modelo pedagógico de AC, seguida de El papel del docente en el aula deEF, las Emociones suscitadas de las prácticas y, por último, la dimensión D esventajas de la aplicación del modelo pedagógico de AC.

\section{Ventajas de la aplicación del modelo pedagógi- co de AC}

Esta dimensión estuvo compuesta por un total de 21 códigos y 215 citas. Tal como se aprecia en laTabla 2, los participantes manifestaron su percepción acerca de las ventajas y beneficios que ofrece el uso del modelo pedagógico de AC en el aula, en concreto, en el área de EF. Además, según los resultados extraídos de las dife-

\begin{tabular}{|c|c|c|}
\hline Dimensión & Códigos & Citas \\
\hline \multirow{21}{*}{$\begin{array}{l}\text { Ventajas de la } \\
\text { aplicación del } \\
\text { Aprendizaje } \\
\text { Cooperativo }\end{array}$} & Beneficios del AC & 23 \\
\hline & Mejoras socioafectivas & 22 \\
\hline & Mejoras emocionales & 20 \\
\hline & Interacción & 16 \\
\hline & Motivación & 15 \\
\hline & Uso del AC positivo & 15 \\
\hline & Autonomía & 13 \\
\hline & Protagonismo & 11 \\
\hline & Fomenta el trabajo de valores & 11 \\
\hline & Aprendizaje entre iguales & 10 \\
\hline & Participación & \\
\hline & Retroalimentación & \\
\hline & $\begin{array}{l}\text { Beneficia a todo el alumnado independientemente de sus capacidades y } \\
\text { per sonalidad }\end{array}$ & \\
\hline & Interviene o col abora en la formación de la per sonal idad del alumnado & 6 \\
\hline & Beneficios iguales para chicosy chicas & 5 \\
\hline & Creatividad & 5 \\
\hline & Razones para aplicar el AC & \\
\hline & Aplicabilidad a todos los cursos & \\
\hline & Alumnos y al umnas aportan iniciativas e ideas & 4 \\
\hline & Demanda del AC por parte del alumnado & 4 \\
\hline & Autoevaluación & 3 \\
\hline
\end{tabular}


rentes entrevistas, esta es la dimensión a la que más importancia otorgaron los entrevistados.

En función de los resul tados obtenidos se puede afirmar que, según los participantes, el uso del AC en el aula es positivo (15 citas), ya que ofrece numerosos beneficios para el alumnado (23 citas). Entre los beneficios más destacados se encuentran las mejoras socioafectivas ( 22 citas) y emocionales (20 citas).

«Pues, yo creo que para el área de EF es muy interesante porque tiene un componente social importante. Bueno, en realidad, todas las áreas deberían tenerlo, pero especialmente nuestra área yo creo que es una de sus partes más fuertes y, este tipo de metodologías bien llevadas puede tener muchas cosas positivas para los alumnos.» (M aestro de EF en EP).

«El AC es positivo porque mejora su autoconcepto, aumenta la motivación, la disminución de conflictos, ayuda a educar en valores, en las habilidades social es y, sobre todo, lo que decíamos antes, que ayuda a paliar algunos conflictos, no todos, pero tienes herramientas con ello para paliar conflictos. Pero, sobre todo, por el aspecto socializador.» (Maestro de EF en EF).

«Es positivo porque dejamos un poquito a un lado la competitividad que, sobre todo en EF desde los primeros cursos ya hay competitividad, y yo creo que mejora mucho las relaciones entre ellos, también dejan a un lado las diferencias que puedan existir, cada uno tiene su nivel de aprendizaje o su ritmo de aprendizaje y entre todos se complementan mucho.» (Maestra de EF en EF).

Como se puede observar en sus respuestas, los entrevistados argumentaron que el uso del $\mathrm{AC}$ es positivo y ofrece diversos beneficios para el alumnado. Dichos argumentos, coinciden con los expuestos por FernándezRío et al. (2014), quienes apuntan que, algunos de los beneficios que aporta este modelo pedagógico, son que aumenta la participación e interacción del alumnado, permite la adquisición de valores y actitudes positivas, asertivas y empáticas hacia los compañeros, ofrece la posibilidad de percibir las situaciones y problemas desde diversas perspectivas, además de que desarrolla la autonomía y la creatividad.

En estalínease encuentrael trabajo deAntón (2016), en el que se midió de manera cuantitativa la relación entre la metodología cooperativa en las clases de EF y la inteligencia emocional con alumnado de 30 y 4을 de Primaria, encontrando una relación entre ambos constructos, y destacando la variable de atención emocional (Antón, 2016), entendida como el grado en que las personas prestan atención a sus sentimientos y emociones. Asimismo, en otra investigación, en la que se aplica un programa de educación emocional cooperativo con al umnado de $1^{0}$ de Primaria, obtuvieron niveles superiores de competencia emocional en los participantes del grupo de intervención con respecto al control (Ambrona, Lopez-Perez y M arquez- Gonzalez, 2012), apoyando de nuevo la tesis inicial acerca de los beneficios del AC y las mejoras socioafectivas y emocionales que de el subyacen.

Con respecto a las mejoras socioafectivas como tal, los entrevistados resaltaron que aparecen como consecuencia del aumento del interés y motivación (15 citas) por parte del alumnado hacia la EF y hacia este tipo de modelos pedagógicos, fomentando un aumento en su participación (ocho citas), así como en la interacción (16 citas) entre los mismos.

«En los registros cuando terminas una sesión, ahí puedes ver si ha mejorado esa motivación que tenemos, esa autoestima [... ]» (Maestro de EF en EP).

"Yo lo llevo usando muchísimo tiempo, muchos años y estas clases de EF, yo creo que les gustan al 99\% .» (M aestra de EF en EP).

«Se puede ver sobre todo a la hora de interactuar con los demás.Yo, por ejemplo, cuando empieza el curso con unos niños, pues los ves que están di stantes, que no quieren moverse, que no quieren relacionarse, y conforme va pasando el tiempo, ves como opinan, como se meten en la clase, quieren participar más, ayudan a sus compañeros, etc. y cuando acaba el curso te das cuenta de que se comportan de una manera totalmente distinta 0 interactúan con los demás de una manera distinta de cómo empezó el curso.» (M zestro de EF en EP).

En el caso de las mejoras emocionales, todos los entrevistados afirmaron que estas mejoras se deben a la cesión de las responsabilidadesal propio alumnado, ofreciéndole mayor libertad y autonomía (13 citas), a la vez que se le da mayor protagonismo (11 citas) en su proceso de enseñanza-aprendizaje.

«Pues observándolos, o sea se sabe perfectamente desde el principio que los conoces y empiezas a trabajar con este tipo de metodol ogías, los alumnos que siempre son mucho más retraídos o que nunca participan porque piensan que son inferiores al resto, conforme les vas dando protagonismo, yo creo que ellos confían cada vez más en sí mismos, y es que los ves, es que los chicos no se quedan atrás, quieren participar y son uno más.» (Maestra de EF en EP).

«Va a fomentar el aspecto socializador o va a fomentar su autonomía o va a fomentar la empatía [... ]» (M zestro de EF en EP).

Estos datos coinciden con la afirmación deVelázquez (2013), quien expone que, mediante el AC se cede al alumnado mayor responsabilidad en la construcción de su propio conocimiento. Del mismo modo, Contreras, Arribas y Gutiérrez (2017), declaran que este modelo 
pedagógico sitúa al alumnado en el centro del proceso, convirtiéndolo en protagonista, tanto de su propio aprendizaje como del de sus compañeros. Al respecto, según los entrevistados, esto se ve reflejado en la forma de ser y de actuar del alumno, afirmando que el modelo de AC interviene o colabora en la formación de la personalidad del alumnado (seis citas).

«Esta metodología sí interviene en la personalidad del al umnado, y además, yo creo que los moldea porque en un futuro se van a encontrar con esta realidad y que la vida no se trata de ir de manera individual, sino quese trata de ir con el resto de personas, entonces si desde pequeños les enseñamos a que hay que trabajar en equipo, que hay que aceptar y entender las opiniones del resto, yo creo que, vamos a conseguir que en un futuro, pues todo esto no sean obstácul os sino que sea parte de la vida.» (Maestra de EF en EP).

«Bueno pues, con esta metodología como con cual quier otra, ya sea buena o mala, todas van a influir en el desarrollo de la personalidad del alumno [... ] pues todo lo comentado ante riormente, va a permitir que su desarrollo de su personalidad sea más completo.» (Maestro de EF en EP).

Asimismo, indicaron que estas mejoras se observan en la retroalimentación (siete citas) y autoevaluación (tres citas) de los propios alumnos.

"Lo vemos a través de los instrumentos de evaluación o la propia autoevaluación del alumnado [... ] lo ves con la evaluación, el feedback, o sea lo estás observando diariamente» (Maestro de EF en EP).

«Pues con la retroalimentación que cada niño obtiene, de ver que juntos, pues consiguen más cosas, juntos son capaces de aprender, que es una ventaja tener a alguien a tu lado con el quehacer cosas, al queenseñarle, del queaprender [... ]» (M aestra de EF en EP).

Con respecto a las razones que empujaron a los entrevistados a aplicar este modelo en sus clases (cinco citas), estaban relacionadas con todos los beneficios descritos anteriormente. Además, mostraron interés por crear un ambiente de aprendizaje entre iguales (10 citas), es decir, de aprendizaje mutuo donde se incentive la creatividad (cinco citas) personal de cadaalumnado y pueda combinarse con la del resto. Siguiendo así, lo expuesto por Baños y Arrayales (2019), quienes recalcan la importancia de diseñar y planificar sesiones de EF donde las metas estén enfocadas hacia la superación de uno mismo y la obtención de la satisfacción por el mero aprendizaje sin la necesidad de compararse con el resto de los compañeros. Sesiones orientadas a la coopera ción, ayuda y consecución del éxito entre un grupo de iguales.

«Pues, lo primero de lo que me di cuenta, como yo de peque ño era una persona bastante torpe, pues desdeel primer momento intenté siempre evitar el: «tú no vales», «tú no juegas» y tal, entonces intenté centrarme en algunos que les costaba un poquito más y a través del AC, el quejuega muy bien, si no coopera con el que no juega tan bien, no va a al canzar el objetivo. Por eso empecé a utilizarla para que, me di cuenta sobre todo que, algunos alumnos que tenían ciertas dificultades enseguida de sistían de la EF y no les gustaba, y a través de esto, pues siempre intento que a quien se le da bien no se aburra e intente ayudar y, el que es un poco menos hábil, se integre en la clase y se sienta eficaz y útil dentro de la actividad y no se desanime.» (Maestro de EF en EP).

«[... ]deben tener libertad para experimentar y así, también fomentar su creatividad que es muy necesaria.» (M aestra de EF en EP).

«Bueno pues, digamos que yo soy una persona, en general, que me gusta ir probando cosas nuevas y diferentes, esta en parte fue una de las razones. Luego, conoces a un compañero que empieza a trabajar este tipo de cosas, también en esa época pues estaba preparando oposiciones, no sé, lo vi interesante porque pienso que el componente social ayuda mucho y compensa esas dificul tades y diferencias de niveles que son tan importantes de compensar en la escuela, realmente.» (Maestro de EF en EP).

Del mismo modo, afirmaron que este modelo pedagógico es de gran importancia porque fomenta el trabajo de valores (11 citas) que ayudan a conseguir todos estos beneficios y es imprescindible enseñarlos desde las primeras edades.

"Sí fomenta el trabajo de valores porque nos ayuda a respe tarnos, nos ayuda a ponernos en el lugar del compañero, y yo creo que cualquier metodología fomenta el aprendizaje en valores, pero yo creo que esta más porque los protagonistas son ellos y si no se ayudan entre ellos, no llegamos al objetivo que queremos conseguir, entonces yo considero que sí.» (M aestra de EF en EP).

Dichas razones se encuentran en relación con las expuestas porVelázquez (2013), quien destaca que al gunos de los motivos que impulsan a los docentes a decantarse por el AC son que favorece el desarrollo de habilidades sociales y actitudes prosociales, permite alcanzar logros afectivos, mejorael autoconcepto, aumenta la motivación hacia la práctica de actividad física, promueve la inclusión, mejora el clima de clase y, sobre todo, origina un mayor grado de satisfacción tanto en el alumnado como en el propio docente.

A tal respecto, López-M ondéjar yTomás (2017) realizaron un estudio en relación al uso de una metodología cooperativa y las habilidades socio-emocionales de los estudiantes universitarios de Educación infantil y Pri- 
maria, cuyos resultados reflejaron una mayor empatía y asertividad entre los miembros del grupo, así como una mayor cohesión y confianza en el logro de acuerdos y en la aceptación de puntos de vista diferentes de los propios, estableciendo por tanto una relación positiva entre el uso de este tipo de metodología cooperativa y la mejora de las habilidades sociales y emocionales.

En este sentido, el área de EF presenta un contexto idóneo para educar en valores, así como el trabajo de las competencias emocionales y sociales (Aguilar, García y Gil del Pino, 2021; Ruiz, 2019). Para ello, es necesa rio implementar modelos como el $A C$, ya que permite trabajar valores y actitudes como la responsabilidad, el esfuerzo, la autosuperación, la justicia, la tolerancia, el compañerismo, la solidaridad, el espíritu de equipo, etc. En consecuencia, se puede afirmar que la aplicación del AC es positiva, ofreciendo sus ben eficiosa todo el al umnado por igual, independientemente de sus capacidades y personalidad (siete citas), no existiendo distinciones entre chicos y chicas (cinco citas).

«Bueno pues, si dentro de las actitudes escolares intentamos en la medida de lo posible la no discriminación por razones de sexo, todos los al umnos y alumnas se tienen que beneficiar de la misma manera, es decir, deben tener los mi smos beneficios, osea que ambos tanto chicas como chicos tienen que alcanzar esos beneficios.» (Maestro de EF en EP).

"Yo lo primero que tengo en mis clases, es la coeducación y no hay distinciones. Es más, nunca hay grupos de chicos 0 de chicas, siemprejuegan mixtosy juntos, por lo que, lo mismo que pueden alcanzar los chicos también lo pueden al canzar las chiCas.» (M aestro de EF en EP).

Además, se trata de un modelo aplicable a todos los niveles educativos (cinco citas) que, por su gran componente motivacional, son los propios alumnos quienes demandan su práctica (cuatro citas), aportando nuevas iniciativas e ideas para enfocarlo (cuatro citas).

«Pues mira, la motivación, sobre todo, ellos te lo demandan diariamente cuando tú has utilizado metodologías cooperativas que a ellos les ha producido una motivación, ellos mismos te piden realizar esas actividades, entonces ahí descubres que ellos están mucho más motivados hacia la práctica deportiva porque te solicitan que hagas la sesión de EF que tantos les gustó.» (Maestro de EF en EP).

"Más de una vez me han dicho: «seño, seño, vamos a hacer un reto, vamos a hacer un reto», osea ellos mismos quieren que tú les plantees retos. Es más, luego ellos también de vienen con retos que quieren hacer de un grupo a otro y demás.» (M aestra deEF en EP).

En definitiva, como afirmaVelázquez (2018), el AC ofrece al alumnado multitud de beneficios tanto a nivel cognitivo, como motriz, social y afectivo-motivacional, convirtiéndose en un excelente recurso para promover la verdadera inclusión del alumnado en las clases de EF

\section{Papel del docente en el aula de EF}

Esta dimensión se compone de un total de cuatro códigos y 43 citas (Tabla 3). En dicha dimensión se ma nifiesta la función del maestro en el aula cuando se aplica el modelo deAC, el trabajo y seguimiento constante que este implica, la multitud de posibilidades que ofrece el área de EF para trabajarlo y, por último, los deseos de formación por parte de los docentes.

Tabla 3.

Códigos pertenecientes a la di mensión del papel del docente en el aula de EF. Dimensión Papel del docente en el aula de Educación Física

\begin{tabular}{lc} 
Códigos & Citas \\
\hline Función del maestro o maestra en el aula & 21 \\
Es necesario trabajo y seguimiento constante & 11 \\
Deseos de formación del docente & 7 \\
Programación de EF muy amplia & 4 \\
\hline
\end{tabular}

La labor del docente pasa de ser un mero transmisor de conocimientos a un planificador de las actividades y supervisor de las interacciones que se producen en el grupo (Fernández-Río et al., 2014). Es decir, debe activar el proceso de enseñanza-aprendizaje a través de sus acciones, las cuales serán, principalmente observar, diagnosticar, responder y evaluar (Fernández-Río y Del Valle, 2016), idea estrechamente relacionadacon la aportada por Fernández-Río (2014) cuando define el AC ha ciendo referencia al rol de co-aprendices del alumnado y del docente. Razonamientossemejantesalas respuestas de los entrevistados, quienes concluyeron que su función en el aula (21 citas) era la siguiente:

«N osotros somos un poquito la guía de los chicos. Es decir, debemos trabajar con ellos, darles unas pautas previamente y guiarles siempre en el proceso.» (Maestra de EF en EP).

«El primer día, un poco les explico en qué consiste el trabajo cooperativo y tal, y a partir deahí, les explico un poco lo que van a hacer, cuál es el objetivo del juego o la tarea, y luego les voy dando una serie de normas para que ell os resuel van la tarea y consigan el objetivo». (M restro de EF en EP).

«H ay que intentar ver dónde tiene cada uno su nivel, ver hasta dónde puede llegar para que ll egue a un nivel en el que no se frustre ni vea que no al canza o que nunca llega a una meta u objetivo. Entonces, cada uno en su nivel ponerles metas alcanzables para que el alumno se motive y se sienta útil». (M aestro de EF en EP).

A pesar de asegurar que el AC ofrece multitud de beneficios, la gran mayoría de los entrevistados comentaron que se trata de un modelo pedagógico que requiere mucha dedicación, trabajo y esfuerzo, así como un seguimiento constante para obtener resultados significativos en el alumnado (11 citas), coincidiendo con estudios que re- 
flejan la necesidad de un trabajo progresivo y constante para una satiffactoria implementación, además de para poder alcanzar todos los beneficios que este modelo proporciona y, evitar así volver de nuevo a lo tradicional, competitivo y excluyente (Fernández-Río et al., 2018; H ortigüela, Hernando, González-Víllora, PastorVicedo y Baena-Extremera, 2020; Pérez-Pueyo y Hortigüela, 2019).

"Si yo ahora mismo me pongo y digo «me han dicho que se trabaja muy bien el $A C$, voy a ponerlo mañana en práctica en mi clase», posiblemente me va a funcional mal, ¿por qué?, porque el trabajo cooperativo implica una serie de hábitos que nuestros alumnos de por sí, de casa no los traen y requiere de mucho trabajo previo para que funcione realmente la clase, entonces digamos que tiene ben eficios, pero es necesaria cierta preparación, ciertas pautas para que funcione y tú veas recompensado eso en los niños, porque no tienen ese tipo de hábitos, y esa forma de trabajar no se tiene habitualmente.» (Maestro de EF en EP).

"Creo que esta metodología tiene muchas posibilidades y beneficios, pero requiere de mucho trabajo y que los resultados seven a largo plazo, es decir, no se ven inmediatamente.» (M aestro de EF en EP).

«El simple hecho de trabajar el AC sin más o el hecho de plantear una tarea en sí, no va a mejorar, ósea no va a cambiar que el que no es nada tolerante, sea más tolerante con el que comete un error o que le guste ayudar.» (M aestro de EF en EP).

Al igual que ocurre con cualquier otro modelo pedagógico, el docente debe conocerlo y dominarlo en todos sus aspectos. Como indica el Decreto 54/ 2014, por el que se establece el currículo de Educación Primaria en la Comunidad Autónoma de Castilla-La M ancha, en concreto en el apartado del área deEF (p. 18816), se muestra la necesidad de la actualización docente. En este sentido, esimprescindiblelaformación permanente del profesorado para el desarrollo de su competencia personal y profesional, permitiéndole actualizarse en contenidos acordes con la sociedad actual y enriqueciendo la asignatura y sus propias prácticas. Aspecto que aparece reflejado en diversas investigaciones como la realizada por León-Díaz, Arija-M ediavilla, M artínez-M uñoz y Santos-Pastor (2020). Es por esta razón que algunos de los entrevistados mostraron su deseo por seguir formándose e indagar en profundidad este modelo pedagógico (siete citas).

«M e gustaría experimentar algo nuevo y mi idea era viendo la bibliografía y buscando en internet, aprovechar este verano para leermeal go máse intentar ponerlo en práctica.» (M aestro de EF en EP).
«N unca terminamos de conocer, yo busco y utilizo muchas cosas.» (Maestra de EF en EP).

Por último, hay que señalar que el área de EF desa rrolla gran variedad de contenidos de manera directa y transversal. En relación con el modelo de $A C$, autores como Velázquez (2015) y Fernández-Río y M éndezGiménez (2016), afirman que se trata de un modelo pedagógico con enormes posibilidades en el ámbito de la EF para el trabajo de cualquier contenido. De acuerdo con estos argumentos, los entrevistados concluyeron que la programación de EF es muy amplia (cuatro citas).

«La programación de EF no es nada cerrada, para mí, es muy abierta. Es más, si viene un inspector y me dice:" «enséñame tus sesiones», le puedo decir: "pues tengo esto, pero hago mil cosas más.» (M restra de EF en EP).

«En estosúltimosaños, he descubierto, porqueyo antes cuando hicela carrera no sabía que me podía dar tantas posibilidades el área en sí para trabajar todos estosaspectosque, para mí, personalmente, creo que son muy importantes para la formación desde niños pequeños.» (Maestro de EF en EP).

Johnson, Johnson y Holubec (1999), consideran que un profesor, partiendo desde cualquier materia y nivel, será capaz de implementar esta metodología. De este modo, González-Martí, García-López y Gutiérrez (2018), aplican esta metodología con un grupo de alumnos en riesgo de exclusión social, y obtienen unos resultados favorables, viéndose reflejados muchos de los beneficios previamente descritos en la dimensión Ventajas en la aplicación del modelo pedagógico de AC.

\section{Emociones suscitadas de las prácticas}

Tal como muestra laTabla 4, los cuatro códigos y sus 42 citas se corresponden con las emociones que priman en las clases de EF de manera general, así como con las experimentadas por el alumnado y el docente tras la práctica del modelo de AC, y su relación con la inteligencia emocional.

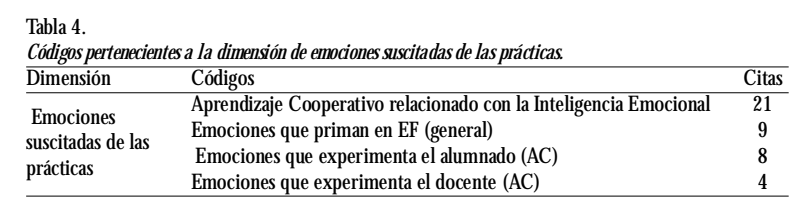

De manera general, el área de EF, dado su carácter práctico y lúdico donde el juego es una herramienta fundamental, produce una mayor manifestación de sentimientos, actitudes y emociones creadas por las rela ciones existentes entre el propio alumnado y el docente (González-Martí, Contreras y Gil-M adrona, 2014). Del mismo modo, como aparece detallado en el De- 
creto 54/ 2014, citado anteriormente, el área de EF es un contexto idóneo para favorecer la adquisición de ha bilidades motrices, mejorar las relaciones sociales y desarrollar la creatividad del alumnado. Además, la diversión, motivación y refuerzos positivos son aspectos fundamentales para generar sentimientos de competencia y confianza en el alumnado, asegurando un clima motivacional adecuado. Sin embargo, es un área que, si no se trabaja de forma adecuada, puede originar conflictos $y$, asociados a ellos, vivencias de emociones y sentimientosnegativas (Aguilar, García y Gil del Pino, 2020). En este aspecto, los entrevistados valoraron la consideración de emociones tanto positivas como negativas en sus clases, aunque estas últimas en menor medida (nueve citas).

«Pues, sobretodo prima la al egría, diversión, la motivación. Entonces, esas son las que me gustan, aunque si es verdad que hay algunas veces que aparece la frustración, el miedo o la impotencia y los niños se agobian un poco, entonces hay que cambiar eso y llevarlo al aspecto al egre, divertido y de bienestar con uno mismo y con sus compañeros.» (Maestro de EF en $E P)$.

«Yo creo que las emociones existen todas. Para mí, el área de EF es perfecta para trabajar las emociones, es decir, puedes ver todo el espectro de emociones en los chicos, desde la mayor ale gría y satisfacción, incluso situaciones de cariño, a situaciones de ira, de frustración total, de miedo, etc. En definitiva, para mí, el área de EF es perfecta para incorporar este componente que creo que es una herramienta brutal en los colegios, más allá del aspecto saludable. Además, entiendo que el trabajo de las emociones influye en la salud [... ]» (Maestro de EF en EP).

En relación con las emociones suscitadas tras la implementación del modelo de AC en sus clases, los entrevistados afirmaron que las emociones experimentadas por el alumnado (ocho citas), cambiaban y, en este caso, surgían en mayor medida las positivas frente a las nega tivas.

«Yo creo que sí que cambian, que priman más las emociones positivas porque ven que trabajando con los demásy ayudándose entre ellos, pueden conseguir lo que se propongan, entonces yo creo que priman las que son más positivas sobre las negativas.» (Maestra de EF en EP).

«Pues, dentro del AC, a lo mejor entran en funcionamiento otras como son la empatía, se fomenta la comunicación porque establecen mucha comunicación entre ellos, el optimismo, solidaridad entreunosy otros, cooperación, comprensión, sobretodo estas, son fundamentales.» (M restro de EF en EP).

De manera similar a como ocurre con el alumnado, se puede afirmar que los maestros también experimentan emociones positivas hacia dicho modelo (cuatro citas).
Igualmente, se puede comprobar en el estudio de Prieto, Fernández-Río, M éndez-Giménezy M éndez (2016), acerca de la percepción del profesorado sobre el AC, que los maestros muestran una actitud positiva, independientemente del área o etapa educativa en la que se encuentren.

«Pues mucha satiffacción porque ves que los niños y niñas están muy motivados, que les gusta y que no resulta al go extraño para ellos este tipo de metodologías.» (Maestra de EF en $E P)$.

«Pues siento mucha satisfacción, sobre todo cuando veo que los niños disfrutan. La mayor satiffacción que yo tengo es cuando parece que no hay maestro de EF en las clases, que ellos se ríen, se al egran, ponen sus normas, no discuten o si lo hacen, ellos mismos se van arreglando [... ]» (Maestro de EF en EP).

Para concluir cabe destacar que, todos los entrevistados, afirmaron que el modelo pedagógico de AC está estrechamente relacionado con la Inteligencia Emocional (21 citas), entendida como la capacidad de reconocer tanto los propios sentimientos como los de los demás, de motivarnos y desarrollar de manera adecuada las rela ciones (Goleman, 1996), ya que fomenta mejoras socioafectivas y emocionales en el alumnado.

"Claro que se relaciona con la Inteligencia Emocional porque si tu cuando estás cooperando te sientes útil, te sientes bien con tus compañeros tus emociones van a estar bien. Además, yo lo trabajo mucho el desarrollo emocional, busco que sea muy Iúdico, divertido, que tengan que hablar, participar y, aunque ahora estamos en un tiempo en el que no se debe y no se va a poder, pues que se abracen mucho, tengan contacto físico, que estrechen lazos emocionales que les ayuden a progresar a lo largo del curso.» (Maestro de EF en EP).

«Un compañero y yo trabajamos juntos en esto y nos planteamoshacer una pequeña ficha deautoevaluación cuando acabas la sesión, pues para que ellos sean capaces de expresarse y decir cómo se sienten, es decir, ¿cómo te sientes antes?, ¿después?, ¿cómo te sientes si tienes sol uciones?, ¿si no tienes?, ¿cómo te sientes si cuentan contigo?, ite has frustrado cuando no te escuchaban?, bueno este tipo de cosas a mí me encantan y la Inteligencia Emocional y poder trabajarla en cualquier aspecto. Este es un componente que enriquece mucho más la actividad cooperativa, que en un principio ya es interesante.» (Maestro de EF en $E P)$.

Además, como exponen Contreraset al. (2017), tanto las emociones, como la autoestima o el estado de ánimo son sentimientos a los que nos enfrentamos cada día y que influyen en nuestro rendimiento. Del mismo modo, Velázquez (2018) afirma que el AC no solo persigue objetivos motores o cognitivos, sino también socia 
les y afectivo-motivacionales.

\section{D esventajas de la aplicación de la metodología de $A C$}

Tal como refleja laTabla 5, esta dimensión está compuesta por un total de cuatro códigos, relacionados con 30 citas, donde se recogen los códigos pertenecientes a las desventajas de laaplicación del AC, la competitividad, los conflictos ocasionados y el castigo establecido.

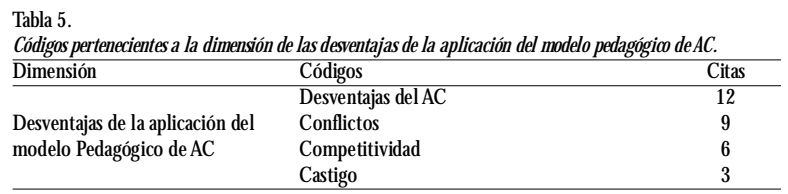

El AC, como se ha descrito anteriormente, tiene efectos muy positivos sobre todo el alumnado, pero no todo es fácil y, cuando no se aplica de manera correcta pueden hallarse ciertas desventajas (12 citas).

«También puede ser que, si el AC no está tomando la dinámica 0 si el niño tiene algún problema, puede cambiar y las emociones positivas se pueden volver en frustración, enfado, odio, a veces ira hacia los propios compañeros o hacia el maestro [... ]» (Maestro de EF en EP).

Algunos problemas, errores o miedos que aparecen en la práctica, según autores como Fernández-Río et al. (2014), pueden ser el incumplimiento de las normas realizando «trampas» en el proceso, críticas o actitudes negativas hacia los propios compañeros de equipo por no haber alcanzado el objetivo, apareciendo la figura del líder o la competición intergrupal. Dichos problemas son muy comunes entre los entrevistados ya que la gran mayoría de estos afirmaron haberlos experimentado en alguna ocasión, destacando el liderazgo y la competitividad (seis citas) como las principales razones por las que surgen la mayoría de conflictos (nueve citas).

«Aparece el liderazgo porque siempre cuando formamos grupos, aunque sean heterogéneos, siempre hay líderes, por lo que surge la función de liderazgo.» (Maestro de EF en EP).

«Siempre hay a lo mejor algún motivo de disputa, de conflicto, pues porque si la culpa es de Fulanito o Fulanita, porque ha salido mal o tal [... ]» (M aestro de EF en EP).

«[... ]por ejemplo, les planteas un reto, ¿no?, ahí habrá niños que por su personalidad y su forma de ser resolverán el problema sin que surja ningún conflicto, y luego te irás al otro grupo y verás que no han propuesto nada, han surgido muchos conflictos, no han sido capaces de hablar, ha surgido el líder [... ]» (Maestro de EF en EP).

En definitiva, retomando la función del docente en el aula, este deberá orientar, supervisar e intervenir, para resolver los posibles conflictos (Contreras et al.,
2017). En este aspecto, los entrevistados aseguraron actuar inmediatamente asignando castigos (tres citas) 0 facilitando estrategias para reconducir la situación.

«Yo sobre todo los conflictos, lo que hago es intentar no sol ucionarlos yo, a no ser que sea un conflicto muy grande.Yo la primera norma que pongo a mis alumnos en mis clases es que, al primer insul to o primera voz, se para la clase, se deja tiempo de reflexión, que dialoguen entre ellos lo que han hecho mal y, hasta que no lo solucionen, no vuel ven a jugar. Si veo que ellos no lo consiguen, pues les voy dando unas pautas, que siguen sin sol ucionarlo, pues seguimos $y$, como ven que van perdiendo clase y selesva el tiempo, al final recapacitan.» (Maestro de EF en $E P)$.

«Si veo que están surgiendo demasiados conflictos y no son capaces de solucionarlo, lo que hago es que una actividad que les gusta mucho, pues se las quito y ellos lo que no quieren es que se las quites.» (Maestra de EF en EP).

\section{Conclusiones}

Los resultados de estainvestigación demuestran que, de manera general, la percepción que posee el profesorado del área de EF en la EP hacia el modelo pedagógico de AC es positiva, independientemente de la edad y experiencia docente. En este caso, se puede afirmar que el modelo ofrece multitud de beneficios tanto para el al umnado como parael docente. Del mismo modo, queda reflejado que el $A C$ está estrechamente relacionado con la Inteligencia Emocional, permitiendo al alumnado obtener mejoras socioafectivas y emocionales que intervienen en el desarrollo de su personalidad. Además, el AC es adecuado para el aprendizaje y adquisición de valores fundamentales para convivir en sociedad. Sin embargo, su implementación y desarrollo no es tarea fácil, y los docentes deben dedicarle mucho esfuerzo y trabajo, además de realizar un constante seguimiento para lograr resultados significativos.

Con respecto a las limitaciones surgidas en el desarrollo de la presente investigación, cabe destacar que, dada la circunstancia en España que da lugar al Real Decreto 463/ 2020, de 14 de marzo, por el que se declaró el estado de alarma para la gestión de crisis sanitaria ocasionada por la Covid-19, la toma de datos tuvo que realizarse de manera online, a través de la plataforma descrita en el apartado de método. De este modo, resultó más complejo lograr el rapport positivo que las entrevistas requieren.

Como prospectiva de investigación se propone la real ización de estudios con una muestra mayor que permita conocer en profundidad la percepción del profeso- 
rado de EF acerca del AC con el fin de poder generalizar los resultados. Además, se podrían incorporar las opiniones del profesorado perteneciente a otros niveles educativos, o procedentes de centros educativos concertados y privados, así como de centros situados en contextos vulnerables.

\section{Referencias}

Aguilar, M., García, C.M., y Gil, C. (2021). Efectividad de un programa educativo en Educación Física para fomentar las habilidades socioafectivas y prevenir la violencia en educación primaria. Retos, 41, 492-501. doi.org/ 10.47197/ retos. v0i41.82683

Antón, R. (2016). La Inteligencia Emocional a traves del AC en las clases deEducacion Física. (Trabajo fin demáster). Universidad de 0 viedo. Recuperado de http:/ / digibuo. uniovi.es/ dspace/ bitstream/ 10651/ 38512/ 6/ TFM RamonAntonGarrido.pdf

Ambrona, T., Lopez, B. y M arquez, M. (2012), Eficacia de un programa de educacion emocional breve para incrementar la competencia emocional de ninos de educacion primaria. Revista Española de Orientación y Psicopedagogía, 23, 39-49. Doi: 10.5944/ reop. vol.23. num.1.2012.11392

Baños, R. , y Arrayales, E. (2019). Predicción del aburrimiento en la educación física a partir del clima motivacional. Retos 38(38), 83-88. Doi: https:/ / doi.org/ 10.47197/ retos. v38i38. 74301

Barrientos, A. (2016). Habilidades sociales y emocionales del profesorado de Educación Infantil relacionados con la gestión del clima de aula (Universidad Complutense deM adrid). Recuperado dehttps:/ / eprints. ucm.es/ 40450/ 1/ T38117.pdf

Bisquerra, R., y Pérez, N. (2007). Las competencias emocionales. Educación XXI, 10, 61-82. Recuperado de: http:/ / e-spacio. uned.es/ fez/ eserv/ bibliuned:EducacionXXI-2007numero10-823/ Documento.pdf

Bisquerra, R., Perez, J. C. y Garcia, E. (2015), Inteligencia emocional en educacion. Madrid, Espana: Sintesis.

Contreras, O. R., Arribas, S., y Gutiérrez, D. (2017). Didáctica de la EF por modelos para Educación Primaria. Madrid: Síntesis.

Decreto 54/ 2014, por el que se establece el currículo de Educación Primaria en la Comunidad Autónoma de Castillat La Mancha. Diario O ficial de CastillatLa Mancha, núm. 132, de 11 de julio de 2014, 1881218846.

Dyson, B. (2001). Cooperative Learning in an
Elementary Physical Education Program. Journal of Teaching in Physical Education, 20(3), 264-281. doi: https:/ / doi.org/ 10.1123/ jtpe.20.3.264

Fernandez-Rio, J. (2003). El AC en el aula de educacion fisica. Analisis comparativo con otros sistemas de ensenanza y aprendizaje. Valladolid: La Peonza.

Fernández-Río, J. (2014). A portaciones del Modelo de Responsabilidad Personal y Social al AC. Actas del IX Congreso Internacional de Actividades Físicas Cooperativas, 18-32. Recuperado de https:// www. researchgate. net/ profile/ Javier_FernandezRio/ publication/263659643_Aportaciones_del Modelo_de_Responsabilidad_Pérso nal_y_Social_al_Aprendizaje_Cooperativo/ links/

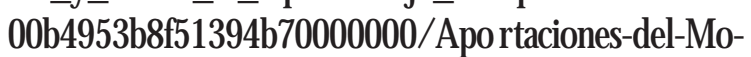
delo-de-Responsabilidad-Personal-y-Social-al-A prendizaje-Cooperativo. pdf

Fernández-Río, J., y Del Valle, P. (2016). El profesor como activador en el AC. Actas del X Congreso Internacional de Actividades Físicas Cooperativas. Recuperado de https:/ / www. researchgate. net/ publication/ 310951795_El_profesor_como_activador _en_el_aprendizaje_cooperativo

Fernández-Río, J., y Méndez-Giménez, A. (2016). El AC: M odelo Pedagógico para la EF. Retos: nuevas tendencias en EF, deporte y recreación, 29, 201-206.

Fernández-Río, J., Calderón, A., Hortigüela, D., PérezPueyo, Á., y Aznar, M. (2016). Modelos pedagógicosen EF: consideraciones teórico-prácticas paradocentes. Revista Española de EF y D eportes, 413, 55-75. Recuperado de https:/ / pdfs.semanticscholar.org/ 6b54/ 6302079c6a0dff6d938a9b72f2bc95b1308a.pdf Fernández-Río, J., Hortigüela, D., y Pérez-Pueyo, Á. (2018). Revisando los modelos pedagógicos en EF. Ideas clave para incorporarlos en el aula. Revista Española de EF y Deportes, 423, 57-80. Recuperado de https:/ / ww w. researchgate. net/ publication/ 328748789_Revisando_los_modelos_ped agogicos_en_educacion_fisica_İeas_clave_para incorporarlos_al_aula

Fernández-Río, J., Rōíguez, J. M., Velázquez, C., y Santos, L. (2014). Actividades y juegos cooperativos para educar en la escuela y en el tiempo libre. Madrid: Editorial CCS.

García, J. A. (2012). La Educación Emocional, su importancia en el proceso de aprendizaje. Revista Educación 36(1), 97-109. Doi: http:/ / dx.doi.org/ $10.15517 /$ revedu.v36i1. 455

Goleman, D. (1996). Inteligencia Emocional. Barcelona: Kairós. 
González-Martí, I., Contreras, 0. R., y Gil-Madrona, P. (2013). Percepción de la competencia emocional en las clases de EF en Educación Primaria. Multiárea: revista de didáctica $6,355-365$.

González-M artí, I., García-López, L. M ., y Gutiérrez, D. (2018). Percepción de una profesora sobre una intervención de $\mathrm{AC}$ con niños en riesgo de exclusión. Actas del XI Congreso Internacional de Actividades Físicas Cooperativas, 391-399. Recuperado de https:/ / pazuela.files. wordpress.com/ 2018/ 10/ libro-congreso-actividades-cooperativas-2018. pdf

Hortigüela, D., Hernando, A., González-Víllora, S. , Pastor-Vicedo, J. C., y Baena-Extremera, A. (2020). «Cooperative Learning does not work for me»: Analysis of its Implementation in Future Physical Education Teachers. Frontiers in Psychology.

Johnson, D. W. y Johnson, R. J. (1999). Aprender juntos y solos.AC, competitivo eindividualista. BuenosAires: Aique S. A.

Johnson, D. W., Johnson, R. T., y Holubec, E. (1999). El $A C$ en el aula. BuenosAires: Paidós.

León-Díaz, Ó ., Arija-M ediavilla, A., Martínez-Muñoz, L., y Santos-Pastor, M. (2020). Las metodologías activas en Educación Física. Una aproximación al estado actual desde la percepción de los docentes en laComunidad deM adrid. Retos38(38), 587-594. Doi: https:/ / doi. org/ 10.47197/ retos. v38i38. 77671

López-M ondéjar, L. M., y Tomás, L. M. (2017). Development of socio-emotional skills through cooperative learning in a niversity environment. Procedia - Social and Behavioral Sciences, 237, 432 437. Doi: 10.1016/ j.sbspro.2017.02.086

Pérez-Pueyo, Á., y Hortigüela, D. (2019). ¿Y si toda la innovación no es positiva en Educación Física? Reflexiones y consideraciones prácticas. Retos 37(37), 579-587. Doi: https:// doi.org/ 10.47197/ retos. v37i37.74176

Pérez, V., Fuentes, J., y Devís, J. (2011). El análisis na rrativo en la EF y el deporte. Movimiento, 17(4),

11-42. Recuperado de https:/ / core.ac.uk/ download/ pdf/ 93038113.pdf

Prieto, J. A., Fernández-Río, J., Cecchini, J. A., MéndezGiménez, A. , y M éndez, D. (2016). Teachers'attitude and perception towards cooperative learning implementation: Influence of continuing training. Teaching andTeacher Education 59, 438-445. Doi: http:/ / dx.doi.org/ 10.1016/ j.tate. 2016.07.020

Real Decreto 126/ 2014, de 28 de febrero, por el que se establece el currículo básico de la Educación Primaria. Boletín 0 ficial del Estado núm. 52, de 1 de marzo de 2014, 19406-19409.

Real Decreto 463/ 2020, de 14 de marzo, por el que se declara el estado de alarma para la gestión de crisis sanitaria ocasionada por el COVID-19. Boletín O ficial del Estado núm. 67, de 14 de marzo de 2020.

Rovegno, I. (2006). Teaching and learning tactical game play at the elementary school level: the role of situated cognition. En N. Wallian, M.P. Poggi, \& M. Musard (Eds.) Co-construire des savoirs: les metiers de I'intervention dans les APSP, (pp.115-126). Besanc'on: Presses universitaries de Franche-Comte.

Ruiz, J. V. (2019). Valores y EF: tejiendo redes desde la acción didáctica. EmásF: revista digital de EF, 57, 5-8.

Velázquez, C. (2010). AC en EF. Fundamentos y aplicaciones prácticas. Barcelona: INDE.

Velázquez, C. (2013). Análisis de la implementación del AC durantela escolarización obligatoria en el área deEF. (U niversidad deValladolid). Recuperado de https:/ / uvadoc. uva. es/ bitstream/ handle/ 10324/ 2823/ TESIS312130521. pdf. pdf; jsessionid=4F2E77A D312B7757BBB5BC54500AA675?sequence $=1$

Velázquez, C. (2015). AC en EF: estado de la cuestión y propuesta de intervención. Retos, (28), 234-239. Recuperado de https:/ / dialnet. unirioja. es/ servlet/ articulo?codigo $=5147772$

Velázquez, C. (2018). El AC en EF: planteamientos teóricos y puesta en práctica. Acción motriz, 20, 7-16. Recuperado de https:/ / dialnet. unirioja. es/ servlet/ articulo?codigo $=6435704$

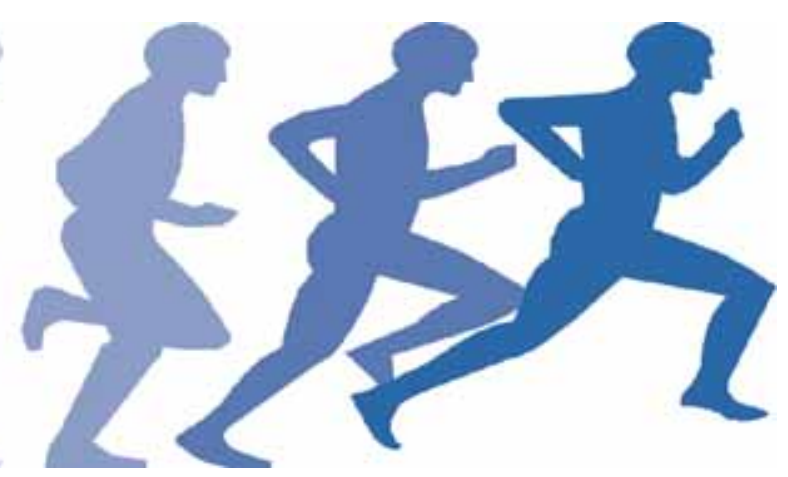

